\title{
Sliding Mode Control of SPMSM Drivers: An Online Gain Tuning Approach with Unknown System Parameters
}

\author{
Jin-Woo Jung ${ }^{*}$, Viet Quoc Leu*, Dong Quang Dang*, Han Ho Choi ${ }^{*}$, and Tae Heoung Kim ${ }^{\dagger}$ \\ *Division of Electronics and Electrical Engineering, Dongguk University, Seoul, Korea \\ $\dagger$ Dept. of Electrical Engineering, Engineering Research Institute, Gyeongsang National University, Jinju, Korea
}

\begin{abstract}
This paper proposes an online gain tuning algorithm for a robust sliding mode speed controller of surface-mounted permanent magnet synchronous motor (SPMSM) drives. The proposed controller is constructed by a fuzzy neural network control (FNNC) term and a sliding mode control (SMC) term. Based on a fuzzy neural network, the first term is designed to approximate the nonlinear factors while the second term is used to stabilize the system dynamics by employing an online tuning rule. Therefore, unlike conventional speed controllers, the proposed control scheme does not require any knowledge of the system parameters. As a result, it is very robust to system parameter variations. The stability evaluation of the proposed control system is fully described based on the Lyapunov theory and related lemmas. For comparison purposes, a conventional sliding mode control (SMC) scheme is also tested under the same conditions as the proposed control method. It can be seen from the experimental results that the proposed SMC scheme exhibits better control performance (i.e., faster and more robust dynamic behavior, and a smaller steady-state error) than the conventional SMC method.
\end{abstract}

Key words: Fuzzy Neural Network Control (FNNC), Sliding Mode Control (SMC), Speed Control, Surface-mounted Permanent Magnet Synchronous Motor (SPMSM), System Parameter Variations

\section{INTRODUCTION}

Permanent magnet synchronous motors (PMSMs) have been gradually replacing both dc motors and induction motors (IMs) in a wide range of drive applications such as electric vehicles, robotic actuators, turbo compressors, etc. This is due to the fact that PMSMs are capable of offering numerous advantages such as high efficiency, low inertia, and high torque-to-current ratio [1], [2] over the aforementioned motors. However, PMSMs have a nonlinear, multivariable, and time-varying system subjected to system uncertainties such as parameter variations and external load torque [3]-[5]. Therefore, it is still challenging to precisely control the speed of PMSMs for achieving a good speed control performance.

Manuscript received Mar. 19, 2014; accepted Jun. 16, 2014

Recommended for publication by Associate Editor Sanjeet K. Dwivedi.

†Corresponding Author: ktheoung@gnu.ac.kr

Tel: +82-55-772-1717, Gyeongsang Nat'1 University

*Division of Electronics and Electrical Engineering, Dongguk University, Korea
Over the past decades, many advanced methods have been presented for the speed control of PMSM systems [4]-[13]. In most papers, the speed controllers are structured by two control terms: a feedback control term and a feedforward control term. First, the feedback control term is designed to make the system dynamics stable. Many researchers are interested in applying the feedback control term into well-known design techniques such as the sliding mode controller [4], [5], feedback linearization controller (FLC) [6], [7], fuzzy logic controller [8], [9], state-dependent Riccati equation controller [10], [11], linear matrix inequality (LMI) controller [12], [13], etc. Meanwhile, the feedforward control term is the main and common term of these controllers, which is employed to compensate for the nonlinear coupling factors using nominal parameters. However, a big disadvantage of these control methods as in [4], [5], [8], [9], and [13] is that the feedforward control term requires full knowledge of the system parameters and load torque conditions with a sufficient accuracy. In general, PMSM systems are always met with unavoidable 
and immeasurable disturbances or some parameter variations. As a result, when the system parameters vary during the drive system operation, there is a mismatch between the nominal and actual values. This can have a strong influence on the control performance of these conventional schemes. Another limitation is that, these control methods [4]-[6], [8]-[11], and [13] also need an assumption that the time derivative of the load torque is equal to zero. This may lead to a slow transient behavior of the load torque observer. Consequently, this makes the speed transient response sluggish. Hence, the two problems mentioned above constrain the steady-state and transient performances of the speed regulations.

To overcome the previously mentioned difficulties, this paper proposes an online gain tuning rule for a robust sliding mode control (SMC) of surface-mounted permanent magnet synchronous motor (SPMSM) drives. The proposed controller consists of two control terms: a fuzzy neural network control (FNNC) term, which approximates the nonlinearity factors, and a sliding mode control (SMC) term, which stabilizes the system dynamics by employing an online tuning rule. Therefore, the proposed SMC method is very robust to the system parameter variations because it does not require any information on the system parameters. In addition, the stability analysis of the proposed control system is described in detail. Finally, comparative experimental results between the proposed SMC scheme and the conventional SMC scheme are shown to verify the high performance (i.e., fast and robust dynamic response, and small steady-state error) of the proposed algorithm.

\section{OnLine-Gain-Tuning Sliding Mode SPeED CONTROLLER DESIGN AND STABILITY} ANALYSIS

\section{A. Error Dynamic Model}

In the $d-q$ rotating reference frame, a mathematical model of the surface-mounted permanent magnet synchronous motor (SPMSM) can be described as:

$$
\begin{aligned}
& \dot{\omega}=k_{1} i_{q s}-k_{2} \omega-k_{3} T_{L}+\zeta_{1}(t) \\
& \dot{i}_{q s}=-k_{4} i_{q s}-k_{5} \omega+k_{6} V_{q s}-\omega i_{d s}+\zeta_{2}(t) \\
& \dot{i}_{d s}=-k_{4} i_{d s}+k_{6} V_{d s}+\omega i_{q s}+\zeta_{3}(t)
\end{aligned}
$$

where $\zeta_{1}(t), \zeta_{2}(t)$, and $\zeta_{3}(t)$ represent lumps of the system uncertainties and $k_{i}(i=1, \ldots, 6)$ signifies the nominal system parameters as:

$$
\begin{aligned}
& k_{1}=\frac{3}{2 J} \frac{60}{\pi} \frac{p}{4} \lambda_{m}, k_{2}=\frac{B}{J}, k_{3}=\frac{1}{2 J} \frac{60}{\pi}, \\
& k_{4}=\frac{R_{s}}{L_{s}}, k_{5}=\frac{\lambda_{m}}{L_{s}}, k_{6}=\frac{1}{L_{s}},
\end{aligned}
$$

$\omega$ is the electrical rotor speed, $i_{d s}$ and $i_{q s}$ are the $d q$-axis stator currents, $V_{d s}$ and $V_{q s}$ are the $d q$-axis stator voltages, $T_{L}$ is the load torque, $p$ is the number of poles, and $R_{s}, L_{s}, J, B$, and $\lambda_{m}$ are the stator resistance, the stator inductance, the rotor equivalent inertia, the viscous friction coefficient, and the magnetic flux, respectively.

Define the angular acceleration $(\beta)$ and the speed error $\left(\omega_{e}\right)$ as:

$$
\begin{aligned}
& \beta=\dot{\omega}=k_{1} i_{q s}-k_{2} \omega-k_{3} T_{L}+\zeta_{1}(t) \\
& \omega_{e}=\omega-\omega_{d}
\end{aligned}
$$

Note that, in many practical applications, the desired speed $\left(\omega_{d}\right)$ is not changed abruptly for short sampling times. Therefore, it can be reasonably supposed that $\dot{\omega}_{d} \approx 0$. Then, the system dynamic model (1) can be rewritten as:

$$
\begin{aligned}
& \dot{\omega}_{e}=\beta \\
& \dot{\beta}=-k_{2} \beta-k_{1} k_{4} i_{q s}-k_{1} k_{5} \omega-k_{1} \omega i_{d s}-k_{3} \dot{T}_{L}+k_{1} k_{6} V_{q s} \\
& \quad+k_{1} \zeta_{2}(t)+\dot{\zeta}_{1}(t) \\
& \dot{i}_{d s}=-k_{4} i_{d s}+\omega i_{q s}+k_{6} V_{d s}+\zeta_{3}(t)
\end{aligned}
$$

In order to satisfy the control design requirements, a new state variable is defined as:

$$
\sigma=\left[\begin{array}{c}
\sigma_{1} \\
\sigma_{2}
\end{array}\right]=\left[\begin{array}{c}
\beta+\eta \omega_{e} \\
i_{d s}
\end{array}\right]
$$

where $\eta>0$ is a design parameter. With this definition, the following theorem can be proved.

Theorem 1: Consider the SPMSM drive system (4). Then the sliding surface (5) guarantees the asymptotic stability of this system.

Proof: By setting $\sigma=\dot{\sigma}=0$ and using the equivalent control method in [14], it can be shown that the sliding mode dynamics restricted to $\sigma=0$ is given by:

$$
\dot{\omega}_{e}=-\eta \omega_{e}
$$

which is asymptotically stable.

Then, from (4) and (5), the following dynamic error system can be obtained:

$$
\dot{\sigma}=B\left[V_{d q s}-f(t)\right]
$$

where

$$
\begin{aligned}
& B=\left[\begin{array}{cc}
k_{1} k_{6} & 0 \\
0 & k_{6}
\end{array}\right], V_{d q s}=\left[\begin{array}{c}
V_{q s} \\
V_{d s}
\end{array}\right], f(t)=\left[\begin{array}{l}
f_{1}(t) \\
f_{2}(t)
\end{array}\right], \\
& f_{1}(t)=\frac{1}{k_{1} k_{6}}\left[\left(k_{2}-\eta\right) \beta+k_{1} k_{4} i_{q s}+k_{1} k_{5} \omega+k_{1} \omega i_{d s}+k_{3} \dot{T}_{L}\right. \\
& \left.-k_{1} \zeta_{2}(t)-\dot{\zeta}_{1}(t)\right] \\
& f_{2}(t)=\frac{1}{k_{6}}\left[k_{4} i_{d s}-\omega i_{q s}+\zeta_{3}(t)\right]
\end{aligned}
$$

Remark 1: Note that, $f(t)$ is a nonlinear term that contains lumps of the system uncertainties $\left(\zeta_{1}(t), \zeta_{2}(t)\right.$, and $\left.\zeta_{3}(t)\right)$ and their time derivatives. In most of the previous speed control methods, $\zeta_{1}(t), \zeta_{2}(t)$, and $\zeta_{3}(t)$, and their time derivatives are commonly ignored and the nominal values of the system parameters $\left(k_{l}, k_{2}, \ldots, k_{6}\right)$ are used to construct the feedforward 
control term regardless of these system uncertainties [4], [5], [8], [9], and [13]. However, when the system parameters change during the operation of the drive system, the system parameters used in these controllers are mismatched with their actual values. Consequently, the system parameter variations can strongly affect the control performance of these conventional schemes. Moreover, most of the previous methods require an assumption that the time derivative of the load torque is equal to zero [4]-[6], [8]-[11], and [13]. Therefore, these two problems limit the accuracy of the speed regulator.

In this paper, a robust intelligent control scheme, which does not require knowledge of the system parameters, is designed to overcome the above mentioned drawbacks. The proposed controller is designed to take the following form:

$$
V_{d q s}=u_{F N N C}+u_{S M C}
$$

where $u_{F N N C}=\left[\begin{array}{ll}u_{F N N C l} & u_{F N N C 2}\end{array}\right]^{T}$ is the FNNC term that approximates the nonlinear term $f(t)$, and $u_{S M C}=\left[u_{S M C l}\right.$ $\left.u_{S M C 2}\right]^{T}$ is the SMC term that stabilizes the system dynamics.

\section{B. FNNC Architecture}

The FNNC system is based on fuzzy logic and neural network concepts. Thus it possesses both of their advantages. Generally, the FNNC consists of one input layer, some hidden layers, and one output layer. In most practical FNNC applications, three- or four-layer structure is widely employed to effectively approximate any input-output mapping. However, it has been suggested in [15] that the problem with the three-layer structure is the fact that its neurons interact with each other globally, which makes it difficult to improve the approximations at one point without weakening it elsewhere. In the four-layer structure, the approximations in different regions can be adjusted independently of each other and the effects of the neurons are isolated. Therefore, in this paper, a FNNC structure with four layers, such as an input layer (layer 1), a membership layer (layer 2), a rule layer (layer 3), and an output layer (layer 4), is adopted as illustrated in Fig. 1.

The signal propagation and basic function of each layer of the FNNC structure are described in the following:

1) The input layer transmits the input linguistic variables $\sigma_{i}$ to the next layer, where $\sigma_{i}$ is an element in the sliding surface $(\sigma)$.

2) The membership layer represents the input values with the following Gaussian membership function because of its easy implementation:

$$
m_{i j}=\exp \left[-\frac{\left(\sigma_{i}-\varphi_{i j}\right)^{2}}{2\left(\kappa_{i j}\right)^{2}}\right], \quad(i=1,2 ; j=1,2, \ldots, l)
$$

where $l$ is the total number of membership functions; and $\varphi_{i j}$ and $\kappa_{i j}$ are the mean and standard deviations of the Gaussian function in the $j$-th term of the $i$-th input variable $\sigma_{i}$ to the

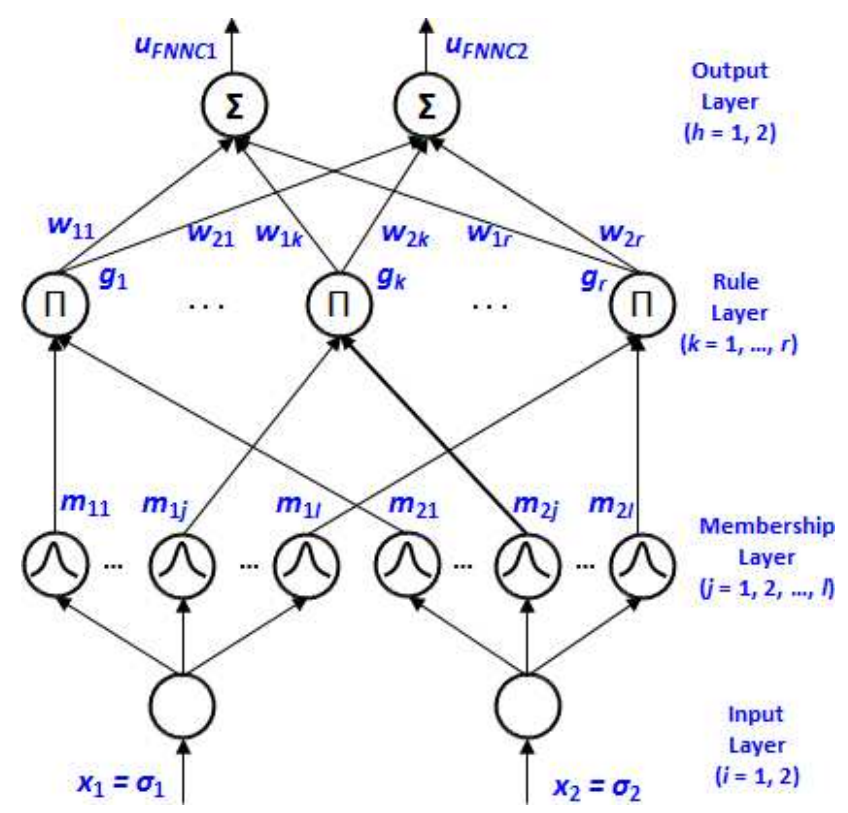

Fig. 1. Configuration of a four-layer FNNC structure.

node of the membership layer, respectively.

3) Each node in the rule layer is denoted by $\Pi$, which multiplies the input signals and generates the result of the product. Thus, the output of this layer is given by:

$$
g_{k}=\prod m_{i j}, k=1, \ldots, r
$$

where $g_{k}$ represents the $k$-th output of the rule layer, and $r$ is the number of the fuzzy rules. In this FNNC structure, $r$ can be calculated by $(l / 2)^{3}$.

4) The final layer is the output layer and its nodes are labeled as $\sum$. This layer computes the total output as the following sum of all the input signals:

$$
u_{F N N C h}=\sum_{k=1}^{r} g_{k} w_{h k}
$$

where $w_{h k}$ is the link weight between the rule layer and the output layer.

It is clear that if the fuzzy rules are increased, the FNNC term will approximate the nonlinear term $f(t)$ more precisely. However, if the number of membership functions used to construct the FNNC term is large, the computational burden may be heavy.

\section{Training Methodology}

In order to train the FNNC term, the energy function is chosen as:

$$
E=0.5 \sigma^{T} \sigma
$$

Thus, the sliding condition is defined as:

$$
\dot{E}=\sigma^{T} \dot{\sigma}<0
$$

From (13), it is guaranteed that $\sigma \rightarrow 0$ as $t \rightarrow 0$. Based on the gradient descent method, the training methodology is derived and it aims to minimize $\sigma^{T} \dot{\sigma}$ to achieve a fast convergence 
of $\sigma$. Therefore, $\sigma^{T} \dot{\sigma}$ is selected as the error function. Then the link weight $\left(w_{h k}\right)$ in the output layer can be updated as:

$$
\begin{aligned}
\dot{w}_{h k} & =-\phi_{h k} \frac{\partial E}{\partial w_{h k}} \\
& =-\phi_{h k} \frac{\partial E}{\partial \sigma_{i}} \frac{\partial \sigma_{i}}{\partial u_{F N N C h}} \frac{\partial u_{F N N C h}}{\partial w_{h k}}=-\phi_{h k} g_{k} \sigma_{i}
\end{aligned}
$$

where $\phi_{h k}>0$ is a learning-rate of the link weight

Let the error term be defined to be propagated as:

$$
\begin{aligned}
\Omega_{j} & =-\sum_{h=1}^{2} \frac{\partial E}{\partial \sigma_{i}} \frac{\partial \sigma_{i}}{\partial u_{F N N C h}} \frac{\partial u_{F N N C h}}{\partial g_{k}} \frac{\partial g_{k}}{\partial q_{i j}} \frac{\partial q_{i j}}{\partial p_{i j}} \\
& =-\sum_{i=1}^{2} \sum_{k=1}^{r} \sigma_{i} w_{h k} g_{k}
\end{aligned}
$$

In the same way as (14), the update rules of $\varphi_{i j}$ and $\kappa_{i j}$ are attained as:

$$
\begin{aligned}
& \dot{\varphi}_{i j}=-\gamma_{1} \frac{\partial E}{\partial \varphi_{i j}}=\gamma_{1} \Omega_{j} \frac{\left(\sigma_{i}-\varphi_{i j}\right)}{\left(\kappa_{i j}\right)^{2}} \\
& \dot{\kappa}_{i j}=-\gamma_{2} \frac{\partial E}{\partial \kappa_{i j}}=\gamma_{2} \Omega_{j} \frac{\left(\sigma_{i}-\varphi_{i j}\right)^{2}}{\left(\kappa_{i j}\right)^{3}}
\end{aligned}
$$

where $\gamma_{1}>0$ and $\gamma_{2}>0$ are the learning-rates of the mean and standard deviations in the Gaussian functions.

\section{Proposed Sliding Mode Speed Control Law with Online Gain Tuning}

By using (11) and (14), the FNNC term can be obtained in the following vector form:

$$
u_{F N N C}=G W, \dot{W}=-\Phi G^{T} \sigma
$$

where $\quad W=\left[\begin{array}{lllllllll}w_{11} & w_{12} & \ldots & w_{1 r} & w_{21} & w_{22} & \ldots & w_{2 r}\end{array}\right]^{T}$ and $\Phi=\operatorname{diag}\left(\phi_{h k}\right)$.

According to the powerful approximation ability of the FNNC [16], there exists an optimal FNNC term $f^{*}(t)$ with an ideal link weight vector $W^{*}$ to learn $f(t)$ such that:

$$
f(t)=f^{*}(t)+\varepsilon=G W^{*}+\varepsilon
$$

where $\varepsilon=\left[\begin{array}{ll}\varepsilon_{1} & \varepsilon_{2}\end{array}\right]^{T}$ is the approximation error and:

$$
\begin{gathered}
G=\left[\begin{array}{cccccccc}
g_{1} & g_{2} & \ldots & g_{r} & 0 & 0 & \ldots & 0 \\
0 & 0 & \ldots & 0 & g_{1} & g_{2} & \ldots & g_{r}
\end{array}\right], \\
W^{*}=\left[\begin{array}{cccccccc}
w_{11}^{*} & w_{12}^{*} & \ldots & w_{1 r}^{*} & w_{21}^{*} & w_{22}^{*} & \ldots & w_{2 r}^{*}
\end{array}\right]^{T}
\end{gathered}
$$

Assume that the approximation error is bounded as $0 \leq\left|\varepsilon_{1}\right|$ $<\rho_{1}$ and $0 \leq\left|\varepsilon_{2}\right|<\rho_{2}$, where $\rho_{1}$ and $\rho_{2}$ are the error bounds. Note that the approximation error can become very small by using more fuzzy rules to construct the FNNC term. However, this option can make the FNNC configuration more complex resulting in a heavy computational burden. Fortunately, the SMC term $\left(u_{S M C}\right)$ can compensate for the difference between the nonlinear term and the FNNC term. It can also guarantee the asymptotic stability of the control system. In this paper, the SMC term is designed as:

$$
u_{S M C}=-\left[\begin{array}{c}
\hat{\rho}_{1} \cdot \operatorname{sgn}\left(\sigma_{1}\right) \\
\hat{\rho}_{2} \cdot \operatorname{sgn}\left(\sigma_{2}\right)
\end{array}\right], \dot{\hat{\rho}}_{1}=\eta_{1}\left|\sigma_{1}\right|, \dot{\hat{\rho}}_{2}=\eta_{2}\left|\sigma_{2}\right|
$$

where $\eta_{1}>0$ and $\eta_{2}>0$ are the learning rates.

Then, the proposed speed control law is obtained by combining the FNNC term and the SMC term as follows:

$$
\begin{aligned}
& V_{d q s}=u_{F N N C}+u_{S M C} \\
& u_{F N N}=G W, \quad \dot{W}=-\Phi G^{T} \sigma \\
& u_{S M C}=-\left[\begin{array}{c}
\hat{\rho}_{1} \cdot \operatorname{sgn}\left(\sigma_{1}\right) \\
\hat{\rho}_{2} \cdot \operatorname{sgn}\left(\sigma_{2}\right)
\end{array}\right], \quad \dot{\hat{\rho}}_{1}=\eta_{1}\left|\sigma_{1}\right|, \dot{\hat{\rho}}_{2}=\eta_{2}\left|\sigma_{2}\right|
\end{aligned}
$$

It is clear that the proposed sliding mode controller is independent of the system parameters because equation (20) does not include any of the system parameter terms. Hence, the proposed control strategy does not require knowledge of the system parameters. Thus, it is very robust to system parameter variations.

Remark 2: By assuming that $\zeta_{1}(t)=\zeta_{2}(t)=\zeta_{3}(t)=0$ and $\dot{T}_{L}=0$ in (4), it is possible to obtain the conventional sliding mode control (SMC) law, which consists of the feedforward control term $\left(u_{F F}=\left[u_{F F 1} u_{F F 2}\right]^{T}\right)$ and the feedback control term $\left(u_{F B}=\left[\begin{array}{ll}u_{F B 1} & u_{F B 2}\end{array}\right]^{T}\right)$, as shown in (21). In this paper, the conventional SMC scheme is employed for a comparative study because it has a control structure similar to that of the proposed SMC method.

$$
\begin{aligned}
& V_{d q s}=u_{F F}+u_{F B}, \\
& u_{F F}=\left[\begin{array}{c}
\frac{1}{k_{1} k_{6}}\left[\left(k_{2}-\eta\right) \beta+k_{1} k_{4} i_{q s}+k_{1} k_{5} \omega+k_{1} \omega i_{d s}\right] \\
\frac{1}{k_{6}}\left(k_{4} i_{d s}-\omega i_{q s}\right)
\end{array}\right], \\
& u_{F B}=-\left[\begin{array}{c}
\lambda_{1} \cdot \operatorname{sgn}\left(\sigma_{1}\right) \\
\lambda_{2} \cdot \operatorname{sgn}\left(\sigma_{2}\right)
\end{array}\right], \lambda_{1}>0, \lambda_{2}>0
\end{aligned}
$$

It is obvious that the above assumptions limit the tracking accuracy of the conventional SMC algorithm (Remark 1). It is important to note that the feedforward control term $\left(u_{F F}\right)$ is structured by the nominal values of the system parameters $\left(k_{l}\right.$ to $k_{6}$ ). Furthermore, the gains of the feedback control term $\left(u_{F B}\right)$ are constant values. Therefore, the performance of the conventional SMC scheme is influenced by parameter uncertainties.

\section{E. Stability Analysis}

Theorem 2: Consider the dynamic error system represented by (7). If the proposed sliding mode controller is designed as (20), then the proposed SMC system is asymptotically stable.

Proof: Let the Lyapunov function $V(t)$ be chosen as:

$$
V(t)=\frac{1}{2} \sigma^{T} G B^{-1} \sigma+\tilde{W}^{T} \Phi^{-1} \widetilde{W}+\frac{\widetilde{\rho}_{1}^{2}}{2 \eta_{1}}+\frac{\widetilde{\rho}_{2}^{2}}{2 \eta_{2}}
$$

where $\tilde{W}=W-W^{*}, \quad \tilde{\rho}_{1}=\hat{\rho}_{1}-\rho_{1}$, and $\tilde{\rho}_{2}=\hat{\rho}_{2}-\rho_{2}$.

The time derivative of $V(t)$ is given by: 


$$
\begin{aligned}
& \dot{V}(t)=\sigma^{T} G B^{-1} \dot{\sigma}+\widetilde{W}^{T} \Phi^{-1} \dot{\widetilde{W}}+\frac{\widetilde{\rho}_{1} \dot{\tilde{\rho}}_{1}}{\eta_{1}}+\frac{\widetilde{\rho}_{2} \dot{\tilde{\rho}}_{2}}{\eta_{2}} \\
& =\sigma^{T} G B^{-1} B\left(V_{d q s}-f(t)\right)+\tilde{W}^{T} \Phi^{-1} \dot{\tilde{W}}+\widetilde{\rho}_{1}\left|\sigma_{1}\right|+\widetilde{\rho}_{2}\left|\sigma_{2}\right| \\
& =\sigma^{T}\left(\left(G W-\left[\begin{array}{l}
\hat{\rho}_{1} \cdot \operatorname{sgn}\left(\sigma_{1}\right) \\
\hat{\rho}_{2} \cdot \operatorname{sgn}\left(\sigma_{2}\right)
\end{array}\right]\right)-\left(G W^{*}+\left[\begin{array}{c}
\varepsilon_{1} \\
\varepsilon_{2}
\end{array}\right]\right)\right) \\
& -\widetilde{W}^{T} \Phi^{-1} \Phi G \sigma+\widetilde{\rho}_{1}\left|\sigma_{1}\right|+\widetilde{\rho}_{2}\left|\sigma_{2}\right| \\
& =-\rho_{1}\left|\sigma_{1}\right|-\rho_{2}\left|\sigma_{2}\right|-\varepsilon_{1} \sigma_{1}-\varepsilon_{2} \sigma_{2} \\
& \leq-\sum_{o=1}^{2}\left(\rho_{o}-\left|\varepsilon_{o}\right|\right)\left|\sigma_{o}\right| \leq 0
\end{aligned}
$$

Since $\dot{V}(t)$ is negative semi-definite, i.e., $V(t) \leq V(0)$, it is concluded from (23) that $\sigma_{1}, \sigma_{2} \in L_{\infty}$. A new term is then defined as:

$$
\vartheta(t) \equiv \sum_{o=1}^{2}\left(\rho_{o}-\left|\varepsilon_{o}\right|\right)\left|\sigma_{o}\right| \leq-\dot{V}(t)
$$

then

$$
\int_{0}^{t} \vartheta(\tau) d \tau \leq V(0)-V(t)
$$

Since $V(0)$ and $V(t)$ are bounded, and $V(t)$ is also non-increasing, the following inequality can be derived:

$$
\lim _{t \rightarrow \infty} \int_{0}^{\infty} \vartheta(\tau) d \tau<\infty
$$

As observed from (23) and (24), $\dot{\vartheta}(t)$ is bounded. Therefore, $\vartheta(t)$ is uniformly continuous. Then the following equation can be obtained by using Barbalat's lemma [16]:

$$
\lim _{t \rightarrow \infty} \vartheta(t)=0
$$

This implies that $\sigma \rightarrow 0$ as $t \rightarrow \infty$. It can be concluded that the dynamic error system (7) with the sliding mode controller (20) is asymptotically stable.

Remark 3: The proposed SMC algorithm (20) requires information on the rotor angular acceleration $(\beta)$ that is usually not available. However, based on the relationship between the rotor speed and acceleration as $\beta=\dot{\omega}$, the estimated rotor acceleration $(\hat{\beta})$ can be simply calculated as [17]:

$$
\hat{\beta}(k)=\frac{T_{o}}{T_{s}+T_{o}} \hat{\beta}(k-1)+\frac{1}{T_{s}+T_{o}}[\omega(k)-\omega(k-1)]
$$

where $T_{s}$ is the sampling time and $T_{o}$ is a filter time that is sufficiently small to control the susceptibility of this calculation to noise.

\section{LABORATORY TEST}

The drive setup of the proposed SMC scheme for SPMSMs is shown in Fig. 2. This drive system consists of a twelve-pole SPMSM, a three-phase VSI, a load motor drive, an encoder, and a control board with a Texas Instruments

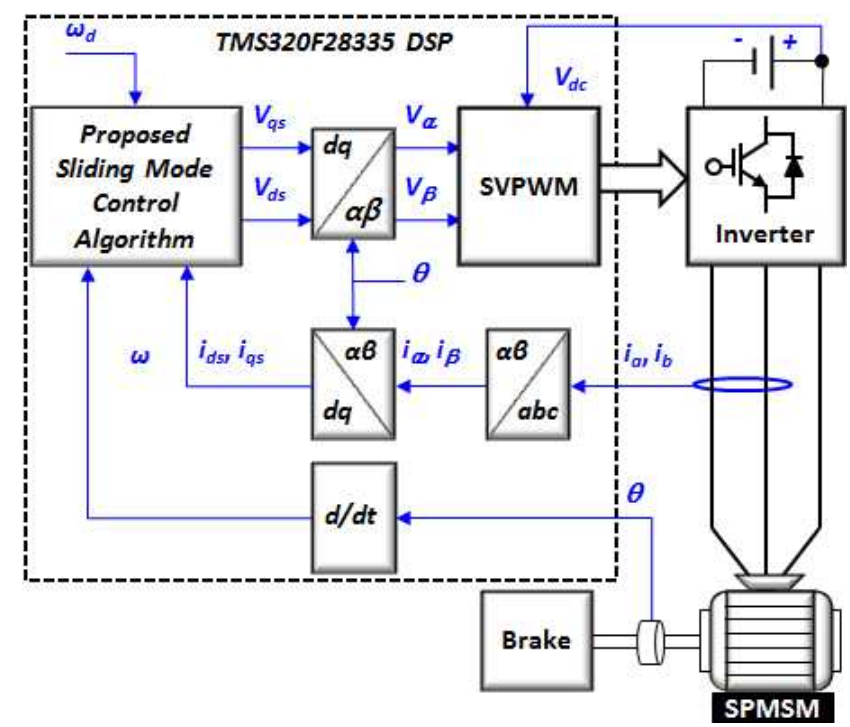

Fig. 2. Overall experimental setup of the SPMSM drive with the proposed sliding mode control scheme.

TABLE I

NOMINAL MOTOR PARAMETERS

\begin{tabular}{|l|c|c|}
\hline Parameter & Symbol & Value \\
\hline Rated power & $P_{\text {rated }}$ & $1 \mathrm{HP}$ \\
\hline Rated stator current & $I_{\text {rated }}$ & $4.3 \mathrm{~A}$ \\
\hline Rated torque & $T_{\text {rated }}$ & $2.4 \mathrm{~N} \cdot \mathrm{m}$ \\
\hline Number of poles & $p$ & 8 \\
\hline Stator resistance & $R_{s}$ & $0.43 \Omega$ \\
\hline Stator inductance & $L_{s}$ & $3.2 \mathrm{mH}$ \\
\hline Magnetic flux & $\lambda_{m}$ & $0.085 \mathrm{~V} \cdot \mathrm{s} / \mathrm{rad}$ \\
\hline Equivalent inertia & $J$ & $0.0018 \mathrm{~kg} \cdot \mathrm{m}^{2}$ \\
\hline Viscous friction coefficient & $B$ & $0.0002 \mathrm{~N} \cdot \mathrm{m} \cdot \mathrm{s} / \mathrm{rad}$ \\
\hline
\end{tabular}

TMS320F28335 DSP. In this system, the rotor position is measured by the encoder, and then the motor speed is easily calculated. For the input signals, only two of the three stator currents are measured by the two Hall-effect sensors and the third stator current can be calculated by equating $i_{a}+i_{b}+i_{c}=$ 0 for a three-phase star connection. Then, the three stator currents are transformed to the two currents $i_{d s}$ and $i_{q s}$ in the $d-q$ rotating reference frame. Finally, the proposed sliding mode controller provides the control inputs $V_{d s}$ and $V_{q s}$, which are transformed into $V_{\alpha}$ and $V_{\beta}$ in the $\alpha-\beta$ stationary reference frame. Note that space vector pulse-width modulation (SVPWM) is utilized to drive the three-phase VSI, which can enhance both the voltage utilization ratio and the efficiency. According to the control performance and overall efficiency, the switching frequency and sampling time $\left(f_{z}\right)$ are selected as $5 \mathrm{kHz}$ and $200 \mu \mathrm{s}$, respectively. Table I lists the nominal parameters of the SPMSM drive.

In this paper, two cases containing a variable load torque and a variable speed are performed to assess the feasibility of 
the proposed control scheme. In addition, motor parameter variations are applied to confirm the robustness of the designed control system. Note that the electrical motor parameters can be changed depending on the temperature and stator currents during the operation of the drive system [19]. In the paper, the motor parameter variations are put as $\Delta R_{s}=$ $+50 \%$ of $R_{s}=0.215 \Omega$, and $\Delta L_{s}=-30 \%$ of $L_{s}=-0.96 \mathrm{mH}$. Therefore, the case studies for experimentation are outlined in Table II.

It is noted that the learning-rates $\left(\phi_{h k}, \eta_{1}\right.$, and $\left.\eta_{2}\right)$ should be sufficiently large to allow for a fast learning process. However, if these values are chosen to be too large, the algorithm may become unstable. Thus, $\phi_{h k}, \eta_{1}$, and $\eta_{2}$ are tuned by taking into consideration the fast learning process and its effectiveness [18]. By referring to (20), $\Phi=500 \cdot I$ and $\eta_{1}=\eta_{2}=100$ are selected. Then, the design parameter in (5) is adopted as $\eta=100$. In addition, the mean and standard deviations of the Gaussian membership functions (9) are chosen as $\varphi_{11}=+300, \varphi_{12}=0, \varphi_{13}=-300, \kappa_{11}=\kappa_{12}=\kappa_{13}=$ $300, \varphi_{21}=+3, \varphi_{22}=0, \varphi_{23}=-3$, and $\kappa_{21}=\kappa_{22}=\kappa_{23}=3$. Moreover, the gains of the conventional sliding method are adopted as $\lambda_{I}=\lambda_{I}=50$. Furthermore, to estimate the rotor angular acceleration $(\beta)$, the sampling time $\left(T_{S}\right)$ and filter time $\left(T_{o}\right)$ are chosen as $T_{s}=1 / f_{z}$ and $T_{o}=T_{s} / 10$, respectively.

First, an experimental study is conducted by considering the variable load torque (Case 1), as shown in Table 2. In this case, the desired speed is set to $300 \mathrm{rpm}$ while the load torque suddenly varies from $0.5 \mathrm{~N} \cdot \mathrm{m}$ to $1.0 \mathrm{~N} \cdot \mathrm{m}$. Figs. 3 and 4 show the experimental waveforms of the proposed SMC scheme for two cases. Each figure shows the desired speed $\left(\omega_{d}\right)$, actual speed $(\omega)$, speed error $\left(\omega_{e}\right), d$-axis stator current $\left(i_{d s}\right)$, and $q$-axis stator current $\left(i_{q s}\right)$. In Fig. 3, the steady-state speed error and the settling time are found to be small (i.e., $6 \mathrm{rpm}$ and $90 \mathrm{~ms}$, respectively). It can be observed that the rotor speed $(\omega)$ can precisely follow the reference value even if the stator resistance $\left(R_{s}\right)$ and stator inductance $\left(L_{s}\right)$ are changed. This means that the proposed control strategy is insensitive to the motor parameter variations when $T_{L}$ changes with a step. Next, an experimental study is performed with a focus on variable speeds (Case 2) and parameter variations. In this case, the desired speed is made to suddenly vary from 300 $\mathrm{rpm}$ to $600 \mathrm{rpm}$ while the load torque is set at $1.0 \mathrm{~N} \cdot \mathrm{m}$. As revealed in Fig. 4, the steady-state speed error is small (8 $\mathrm{rpm})$, and the speed dynamic response is fast with a short settling time $(60 \mathrm{~ms})$. It can be confirmed from Figs. 3 and 4 that the proposed SMC system can accomplish accurate and fast speed tracking despite system parameter variations.

For a comparative evaluation, the conventional SMC scheme described in Remark 2 is also tested under the same conditions as the proposed scheme and the results are given in Figs. 5 and 6. In Fig. 5, the steady-state speed error and settling time are observed to be $20 \mathrm{rpm}$ and $130 \mathrm{~ms}$,
TABLE II

CASE STUDies OF EXPERIMENTATION

\begin{tabular}{|c|c|c|c|}
\hline Cases & Conditions & Details & $\begin{array}{l}\text { Parameter } \\
\text { variations } \\
\end{array}$ \\
\hline 1 & $\begin{array}{l}\text { Variable load } \\
\text { torque }\end{array}$ & $\begin{array}{c}\omega_{d}=300 \mathrm{rpm} ; \\
T_{L}: 0.5 \mathrm{~N} \cdot \mathrm{m} \rightarrow \\
1.0 \mathrm{~N} \cdot \mathrm{m} ;\end{array}$ & \multirow{2}{*}{$\begin{array}{c}\Delta R_{s}=+50 \% \text { of } \\
R_{s} ; \\
\Delta L_{s}=-30 \% \text { of } \\
L_{s}\end{array}$} \\
\hline 2 & $\begin{array}{c}\text { Variable rotor } \\
\text { speed }\end{array}$ & $\begin{array}{c}\omega_{d}: 300 \mathrm{rpm} \rightarrow \\
600 \mathrm{rpm} ; \\
T_{L}=1 \mathrm{~N} \cdot \mathrm{m} ;\end{array}$ & \\
\hline
\end{tabular}

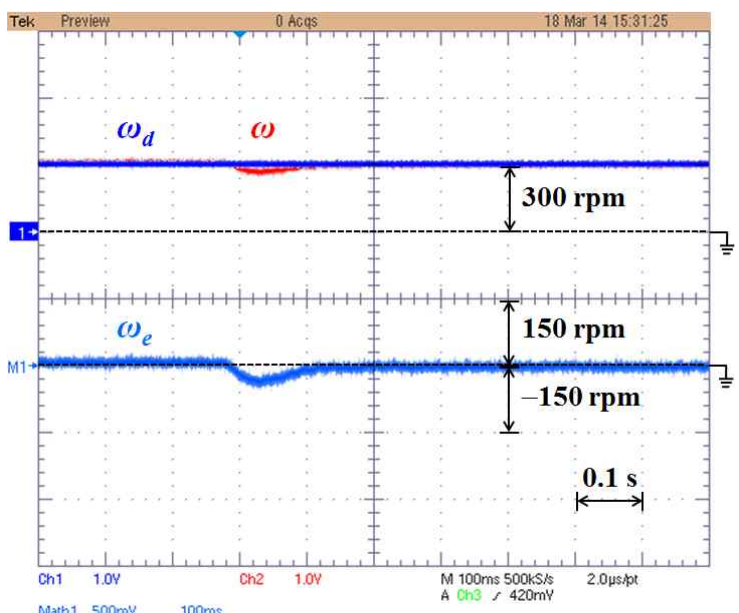

(a)

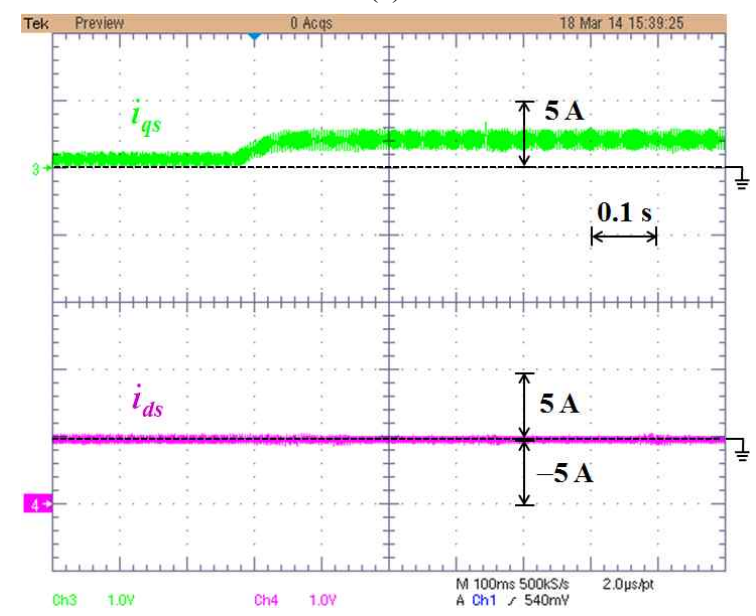

(b)

Fig. 3. Experimental results of the proposed SMC scheme under Case 1. (a) Desired speed $\left(\omega_{d}\right)$, speed $(\omega)$, and speed error $\left(\omega_{e}\right)$. (b) $q$-axis stator current $\left(i_{q s}\right)$ and $d$-axis stator current $\left(i_{d s}\right)$.

respectively. Moreover, Fig. 6 shows that the steady-state error $(16 \mathrm{rpm})$ and settling time $(85 \mathrm{~ms})$ are much larger when compared to those of the proposed control method shown in Fig. 4. Therefore, it can be concluded that when the system parameters vary during drive system operation, the conventional SMC scheme has a mismatch between the nominal and actual values, which highly degrades the control performance. Based on the above analysis, Table III sums up the quantitative results of the two control methods. 


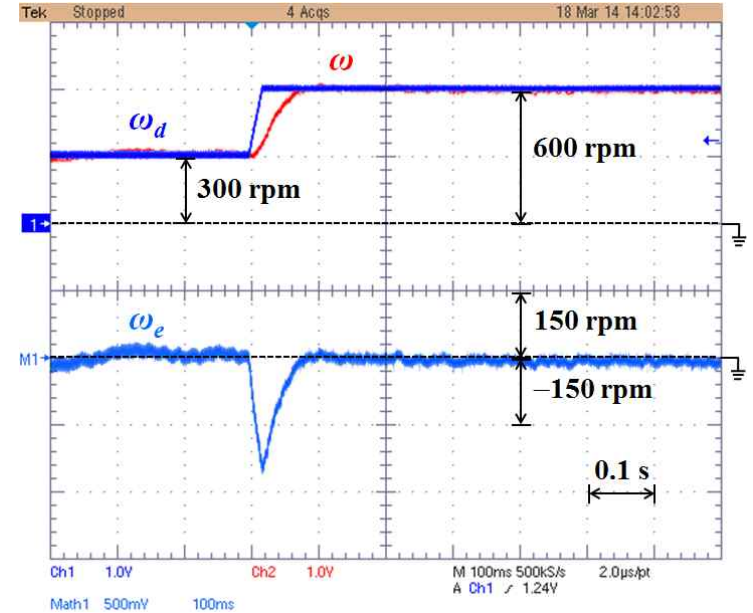

(a)

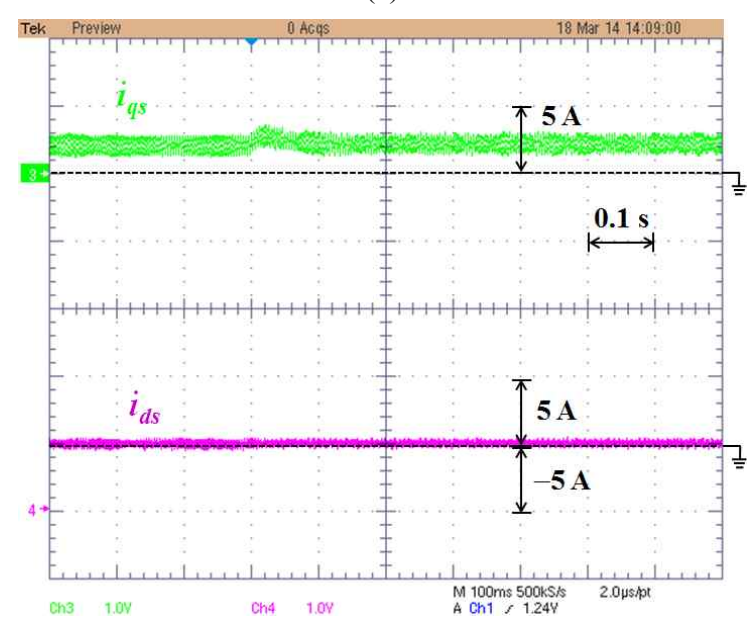

(b)

Fig. 4. Experimental results of the proposed SMC scheme under Case 2. (a) Desired speed $\left(\omega_{d}\right)$, speed $(\omega)$, and speed error $\left(\omega_{e}\right)$. (b) $q$-axis stator current $\left(i_{q s}\right)$ and $d$-axis stator current $\left(i_{d s}\right)$.

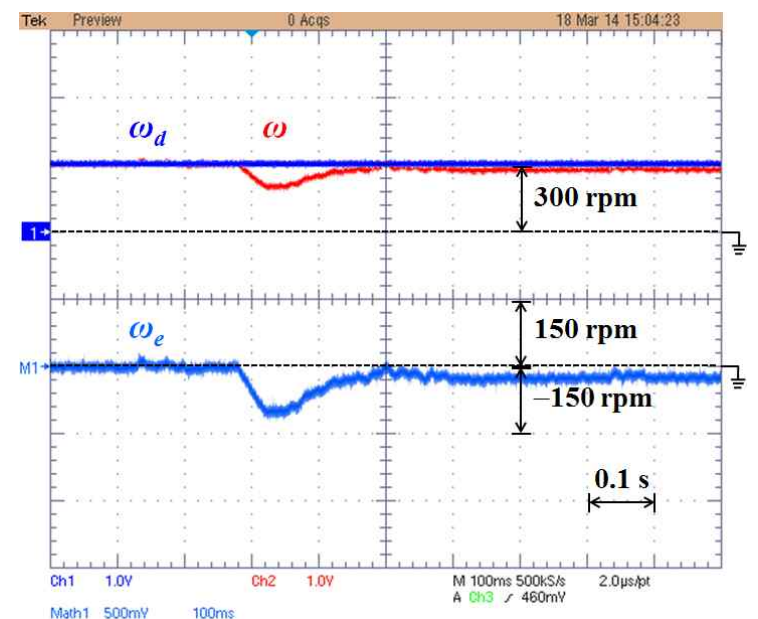

(a)

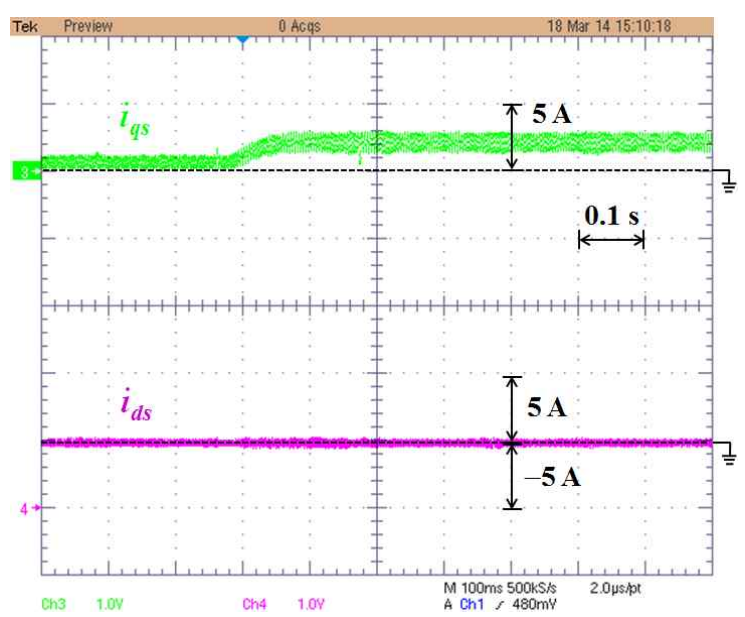

(b)

Fig. 5. Experimental results of the conventional SMC scheme under Case 1. (a) Desired speed $\left(\omega_{d}\right)$, speed $(\omega)$, and speed error $\left(\omega_{e}\right)$. (b) $q$-axis stator current $\left(i_{q s}\right)$ and $d$-axis stator current $\left(i_{d s}\right)$.

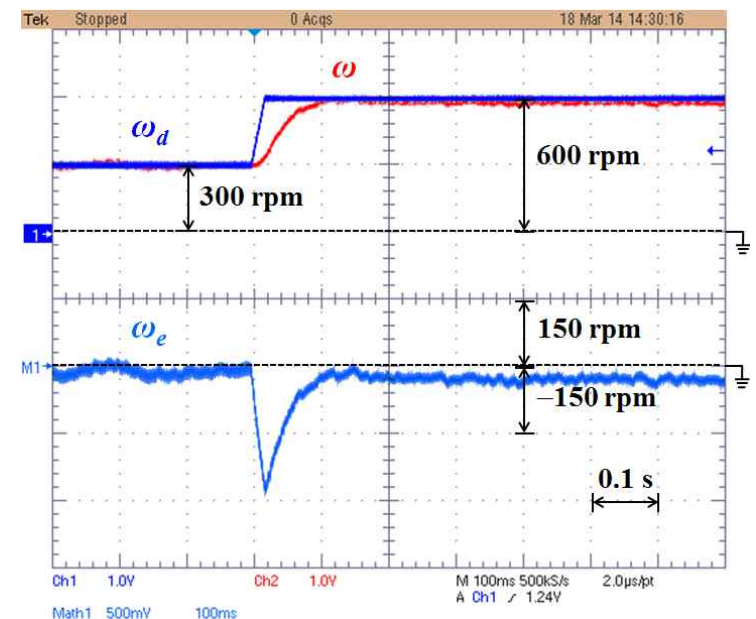

(a)

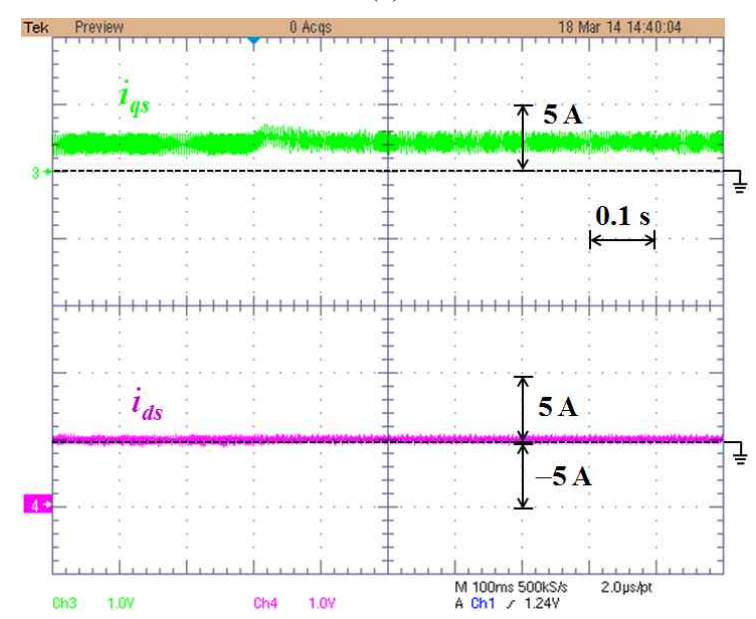

(b)

Fig. 6. Experimental results of the conventional SMC scheme under Case 2. (a) Desired speed $\left(\omega_{d}\right)$, speed $(\omega)$, and speed error $\left(\omega_{e}\right)$. (b) $q$-axis stator current $\left(i_{q s}\right)$ and $d$-axis stator current $\left(i_{d s}\right)$. 
TABLE III

SuMMARY COMPARISONS OF TwO CONTROL AlgORITHMS

\begin{tabular}{|c|c|c|c|c|}
\hline Conditions & Settling & ne $(\mathrm{ms})$ & Steady & te error \\
\hline $\begin{array}{l}\text { Control } \\
\text { algorithms }\end{array}$ & Case 1 & Case 2 & Case 1 & Case 2 \\
\hline $\begin{array}{c}\text { Proposed SMC } \\
\text { scheme }\end{array}$ & 90 & 60 & 6 & 8 \\
\hline $\begin{array}{l}\text { Conventional } \\
\text { SMC scheme }\end{array}$ & 130 & 85 & 20 & 16 \\
\hline
\end{tabular}

The experiments and quantitative results given in Figs. 3-6 and Table III make it clear that the proposed SMC control algorithm provides better speed tracking capability and guarantees more robust tracking against motor parameter variations and load torque disturbances than the conventional SMC method.

\section{CONCLUSIONS}

This paper proposed a robust sliding mode speed controller for SPMSM drives to achieve fast and precise speed tracking. By combining a FNNC term and an adaptive SMC term, the proposed controller did not require any knowledge of the nominal system parameters. Therefore, it was very robust to system parameter variations. Based on Lyapunov theories and related lemmas, a stability evaluation of the proposed SMC system was presented in detail. For this comparison, both the proposed SMC control scheme and the conventional SMC scheme were simulated and tested when the load torque or the desired speed suddenly changes with a step under motor parameter variations. Through these experimental results, it was verified that the proposed SMC method achieved superior control performance (i.e., faster and more robust dynamic behavior and smaller steady-state error) when compared to the conventional SMC method.

\section{ACKNOWLEDGMENT}

This work was supported by the National Research Foundation of Korea (NRF) grant funded by the Korea government (MSIP) (No. 2012R1A2A2A01045312).

\section{REFERENCES}

[1] J. W. Jung, H. H. Choi, and D. M. Lee, "Implementation of a robust fuzzy adaptive speed tracking control system for permanent magnet synchronous motors," Journal of Power Electronics, Vol. 12, No. 6, pp. 904-911, Nov. 2012.

[2] H. S. Kang, C. K. Kim, and Y. S. Kim, "Position control for interior permanent magnet synchronous motors using an adaptive integral binary observer," J. Electr. Eng. Technol., Vol. 4, No. 2, pp. 240-248, Jun. 2009.

[3] K. Hartani, Y. Miloud, and A. Miloudi, "Improved direct torque control of permanent magnet synchronous electrical vehicle motor with proportional-integral resistance estimator," J. Electr. Eng. Technol., Vol. 5, No. 3, pp. 451-461, Sep. 2010.

[4] V. Q. Leu, H. H. Choi, and J. W. Jung, "LMI-based sliding mode speed tracking control design for surface-mounted permanent magnet synchronous motors," J. Electr. Eng. Technol., Vol. 7, No. 4, pp. 513-523, Jul. 2012.

[5] V. Q. Leu, H. H. Choi, and J. W. Jung, "Fuzzy sliding mode speed controller for PM synchronous motors with a load torque observer," IEEE Trans. Power Electron., Vol. 27, No. 3, pp. 1530-1539, Mar. 2012.

[6] J. Solsona, M. I. Valla, and C. Muravchik, "Nonlinear control of a permanent magnet synchronous motor with disturbance torque estimation," IEEE Trans. Energy Convers., Vol. 15, No. 2, pp. 163-168, Jun. 2000.

[7] G. Zhu, L. A. Dessaint, O. Akhrif, and A. Kaddouri, "Speed tracking control of a permanent-magnet synchronous motor with state and load torque observer," IEEE Trans. Ind. Electron., Vol. 47, No. 2, pp. 346-355, Apr. 2000

[8] J. W. Jung, H. H. Choi, and T. H. Kim, "T-S fuzzy tracking control of surface-mounted permanent magnet synchronous motors with a rotor acceleration observer," Journal of Power Electronics, Vol. 12, No. 2, pp. 294-304, Mar. 2012

[9] J. W. Jung, H. H. Choi, and T. H. Kim, "Fuzzy PD speed controller for permanent magnet synchronous motors," Journal of Power Electronics, Vol. 11, No. 6, pp. 819-823, Nov. 2011

[10] H. Beikzadeh and H. D. Taghirad, "Nonlinear sensorless speed control of PM synchronous motor via an SDRE observer-controller combination," IEEE Conf. Ind. Electron. Appl., pp. 3570-3575, May 2009.

[11] P. V. Medagam, T. Yucelen, and F. Pourboghrat, "Adaptive SDRE-based nonlinear sensorless speed control for PMSM drives," Amer. Control Conf., pp. 3866-3871, Jun. 2009.

[12] K. Y. Lian, C. H. Chiang, and H. W. Tu, "LMI-based sensorless control of permanent-magnet synchronous motors," IEEE Trans. Ind. Electron., Vol. 54, No. 5, pp. 2769-2778, Oct. 2007.

[13] H. H. Choi, J. W. Jung, and T. H. Kim, "Digital speed regulator system design for a permanent magnet synchronous motor," J. Electr. Eng. Technol., Vol. 7, No. 6, pp. 911-917, Nov. 2012.

[14] V. Utkin, "Variable structure systems with sliding modes," IEEE Trans. Autom. Control, Vol. 22, No. 2, pp. 212-222, Apr. 1977.

[15] D. L. Chester, "Why two hidden layers are better than one," Int. Joint Conf. Neural Networks, Vol. 1, pp. 265-268, 1990.

[16] J. J. E. Slotine and W. Li, Applied Nonlinear Control, Prentice Hall, 1991.

[17] H. H. Choi, N. T. T. Vu, and J. W. Jung, "Digital implementation of an adaptive speed regulator for a PMSM," IEEE Trans. Power Electron., Vol. 26, No. 1, pp. 3-8, Jan. 2011

[18] M. Moreira and E. Fiesler, "Neural networks with adaptive learning rate and momentum terms," IDIAP Technical Report, Oct. 1995.

[19] F. Fernandez-Bernal, A. Garcia-Cerrada, and R. Fuare, "Determination of parameters in interior permanent-magnet synchronous motors with iron losses without torque measurement," IEEE Trans. Ind. Appl., Vol. 37, No. 5, pp. 1265-1272, Sep./Oct. 2001. 


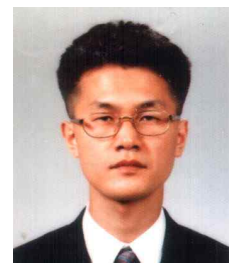

Jin-Woo Jung received his B.S. and M.S. degrees in Electrical Engineering from Hanyang University, Seoul, Korea, in 1991 and 1997, respectively. He received his Ph.D. degree in Electrical and Computer Engineering from The Ohio State University, Columbus, Ohio, USA, in 2005. From 1997 to 2000, he was with the Home Appliance Research Laboratory, LG Electronics Co., Ltd., Seoul, Korea. From 2005 to 2008, he worked as a Senior Engineer at the R\&D Center and with the PDP Development Team, Samsung SDI Co., Ltd., Korea. Since 2008, he has been an Associate Professor with the Division of Electronics and Electrical Engineering, Dongguk University, Seoul, Korea. His current research interests include DSP-based electric machine drives, distributed generation systems using renewable energy sources, and power conversion systems and drives for electric vehicles (EVs).

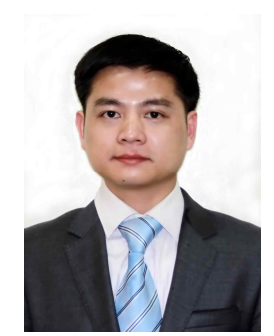

Viet Quoc Leu received his B.S. and M.S. degrees in Electrical Engineering from Hanoi University of Science and Technology (HUST), Hanoi, Vietnam, in 2006 and 2008, respectively, and his Ph.D. degree from the Division of Electronics and Electrical Engineering, Dongguk University, Seoul, Korea, in 2013. His current research interests include DSP-based electric machine drives and control of distributed generation systems using renewable energy sources.

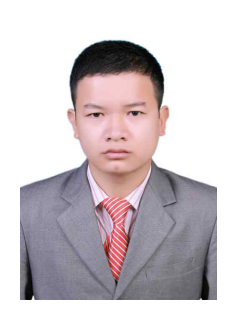

Dong Quang Dang received his B.S. and M.S. degrees in Electrical Engineering from Hanoi University of Science and Technology (HUST), Hanoi, Vietnam, in 2005 and 2010, respectively. From 2006 to 2011, he worked as a Lecturer at Hung Yen University of Technology and Education, Hung Yen, Vietnam. He is currently pursuing his Ph.D. degree in the Division of Electronics and Electrical Engineering, Dongguk University, Seoul, Korea. His current research interests include electric machine drives and control of distributed generation systems using renewable energy sources.

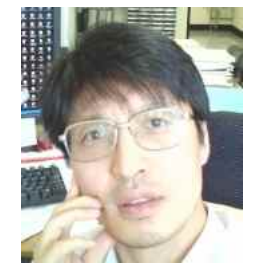

Han Ho Choi received his B.S. degree in Control and Instrumentation Engineering from Seoul National University (SNU), Seoul, Korea, in 1988, and his M.S. and $\mathrm{Ph} . \mathrm{D}$. degrees in Electrical Engineering from the Korea Advanced Institute of Science and Technology (KAIST), Daejeon, Korea, in 1990 and 1994, respectively. He is currently with the Division of Electronics and Electrical Engineering, Dongguk University, Seoul, Korea. His current research interests include control theory and its application to real world problems.

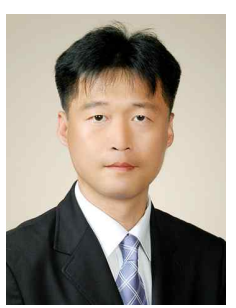

Tae Heoung Kim received his B.S., M.S., and $\mathrm{Ph}$. D. degrees in Electrical Engineering from Hanyang University, Seoul, Korea, in 1993, 1995, and 2005, respectively. From 1995 to 2002, he worked as a Senior Research Engineer for LG Electronics, Korea. Since 2005, he has been a professor in the Department of Electrical Engineering at Gyeongsang National University, Jinju, Korea. His current research interests include the design of electric machines and control systems. 